\title{
Cognición social en la violencia de pareja: una perspectiva neurocriminológica*
}

\section{Social cognition in partner violence: a neurocriminological perspective}

\author{
Wilson Miguel Salas-Picón \\ Universidad Cooperativa de Colombia \\ ORCID 0000-0003-1458-6770 \\ Ibeth Rocío Cáceres Durán** \\ Unidad de Víctimas, Defensoría \\ del Pueblo, Colombia
}

Recibido: 25 de febrero de 2016

Revisado: 30 de marzo de 2016

Aceptado: 1 de mayo de 2016

\section{Resumen}

La evaluación e interpretación que realizan los seres humanos de estímulos reales o imaginados, constituye uno de los factores predictores que determinará el repertorio conductual, ya sea adaptativo o desadaptativo, con alcance jurídico a ejecutar en la relación de pareja. Por lo anterior, el estudio evaluó el desempeño en tareas de cognición social en un grupo de hombres que poseen antecedentes por violencia de pareja. A los participantes se les aplicó pruebas de reconocimiento emocional y empatía. Los resultados indicaron que el grupo de hombres maltratadores presenta dificultades en su cognición social, debido posiblemente a un sesgo intencional en el reconocimiento de las conductas de su congénere, por lo cual, atribuyen una intencionalidad negativa al comportamiento del otro. Estos resultados permiten aportar una base empírica desde la psicología jurídica en la comprensión bimodal de la violencia de pareja, y de esta forma centrar la intervención en las necesidades del individuo.

Palabras clave: violencia de pareja, cognición social y psicología jurídica.

Artículo de investigación. DOI: http://dx.doi.org/10.15332/s1794-9998.2017.0002.10

** Correspondencia: Wilson Miguel Salas-Picón, magíster en Psicología Jurídica, psicólogo, profesor programa de Psicología de la UCC-Barrancabermeja. Dirección postal: calle 60 No. 28-129, Paseo de la Cultura, Barrancabermeja-Santander, Colombia. Correo electrónico: wilsonsalas2@ gmail.com. Ibeth Rocío Cáceres Durán, magíster en Psicología Jurídica, especialista en Psicología Forense, psicóloga. Profesional especializada, Unidad de Víctimas de la Defensoría del Pueblo. Correo electrónico: ibrocadu@hotmail.com. 


\section{Abstract}

Evaluation and interpretation of real or imagined stimuli done by humans is one of the predictors that will determine the behavioral repertoire, be it adaptive or maladaptive, with legal effect to be executed in the partner relationship. Therefore, this study assessed the performance on social cognition tasks in a group of men with a history of intimate partner violence. Participants were given emotion recognition and empathy tests. Results indicate that male abusers have difficulties with social cognition, possibly due to an intentional bias in recognizing the behaviors of others, which results in the attribution of a negative intentionality to their behavior. These results provide an empirical basis for legal psychology in the bimodal understanding of partner violence and thus guides intervention toward the needs of the individual.

Keywords: legal psychology, social cognition, partner violence.

\section{Introducción}

La Organización Mundial de la Salud (OMS) (2002) define la violencia de pareja como un subtipo de violencia intrafamiliar, que comprende el conjunto de comportamientos y acciones emprendidas por los hombres maltratadores hacia sus parejas. Otros autores la definen como un patrón de comportamientos agresivos y coercitivos que presentan los adultos hacia su compañera o compañero íntimo (Jouriles, McDonald, Norwood \& Ezell, 2001; citado por Olaya, Tarragona, De la Osa y Ezpeleta, 2008). En el Informe mundial sobre la violencia y la salud (2002), se define como:

El uso intencional de la fuerza o el poder físico, de hecho o como amenaza, contra uno mismo, otra persona o un grupo o comunidad, que cause o tenga muchas probabilidades de causar lesiones, muerte, daños psicológicos, trastornos del desarrollo o privaciones. (Organización Mundial de la Salud, 2002, p.3)

Por su parte, en el marco jurídico colombiano, Ley 599 de 2000 (en adelante Código Penal), se define que la violencia intrafamiliar se concibe como el maltrato físico, psíquico o sexual, ejercido hacia cualquier miembro de su núcleo familiar.

Las investigaciones sobre violencia intrafamiliar y violencia de pareja (Carreño, 2011), dirigidas principalmente por el Instituto Nacional de Medicina Legal y Ciencias Forenses (2015) y Barón (2010), señalan que la principal forma de violencia intrafamiliar es aquella emitida por la pareja, con un $64,33 \%$, y las cifras sobre violencia de pareja advierten que el $77,58 \%$ de las mujeres que han convivido con sus parejas, sufrieron cierto tipo de violencia; actos que traen consigo costos económicos para el país, puesto que este fenómeno cuesta el $4 \%$ del gasto del producto interno bruto (PIB) (Ribero y Sánchez, 2004; Barón, 2010). Es de aclarar, que si bien es cierto los estudios adelantados dan cuenta de la violencia ejercida mayoritariamente de hombres hacia mujeres, la violencia de pareja también es practicada entre parejas homosexuales (Burke \& Follingstad, 1999; Burke, Jordan \& Owen, 2002) y de mujeres hacia hombres, aunque en menor proporción, lo que desconoce cifras y procesos judiciales adelantados (Morse, 1995; Straus, 1993; Straus y Gelles, 1988; Bartholomew, Regan, Oram y White, 2008; Burke \& Follingstad, 1999; Burke et al., 2002; Turell, 2000). Lo expuesto permite plantear que existen conductas con alcance jurídico, donde la perspectiva normativa resulta insuficiente para la comprensión y prevención de la criminalidad, puesto que el delito constituye un conflicto multicausal, en el cual se involucran las personas en su relación intersubjetiva, en un contexto social determinado (Henry \& Plemmons, 2012; Greely, 2008; Procuraduría General de la República, República Dominicana, 2005).

Las consideraciones descritas previamente, las altas cifras de violencia dentro del núcleo familiar, las incongruencias entre el deber ser desde la legislación con el actuar de la población y el sinnúmero de actuaciones que se han adelantado para mitigar 
la problemática, permiten considerar la violencia de pareja como uno de los principales problemas sociales y de salud pública (Amor, Echeburua y Loinaz, 2009; Amor, Bohórquez y Echeburúa, 2006; Olaya et al., 2008; Krug, Dahlberg, Mercy, Zwi \& Lozano, 2002). La anterior panorámica ha permitido el surgimiento de posturas y perspectivas a partir del análisis multicausal y polirelacional sobre las variables psicológicas y neuropsicológicas, que brindan una amplitud sobre su comprensión y estrategias de abordaje, al tener en cuenta las necesidades de cada individuo.

En consonancia con lo expuesto, el abordaje neurocognitivo, neuropsicológico, neuroemocional y neurosocial de la actividad psicológica en las interacciones humanas, ha tenido un desarrollo importante en los últimos años debido a las contribuciones de las neurociencias, especialmente la neuropsicología social, la cual busca elaborar hipótesis congruentes con las anormalidades en la conducta social, y se centra principalmente en aquellos procesos interpersonales denominados como cognición social, en los que se incluye: la empatía, la simpatía, el razonamiento moral, la regulación y procesamiento emocional, esto con el fin de establecer las representaciones cerebrales y la forma en que estas se manifiestan en la interacción social (Decety, 2010; Decety, 2008; Decety y Meyer, 2008; Urrego, 2011; Uribe, 2010). Por lo anterior, el funcionamiento neuropsicológico del hombre maltratador ha llamado el interés de diversos campos de conocimiento, en el que se destaca la criminología como una subárea de la psicología jurídica. Los trabajos neurocriminológicos realizados sobre la cognición social y el procesamiento emocional de hombres maltratadores son escasos.

La cognición social (CS) puede ser definida como un proceso neurobiológico (Butman, 2001), psicológico y social, mediante el cual es posible percibir, reconocer y evaluar los eventos sociales, y con ello llegar a construir una representación del ambiente en el que interactúan los individuos (Adolphs, 2001), y a partir de ello, plantear el comportamiento social, el cual hace referencia a la respuesta dada por el individuo frente a una circunstancia particular. La cognición social se encuentra relacionada con la percepción social (la cual se presenta como un estadio inicial que evalúa las intenciones de los demás a través de su conducta, por medio de la dirección de la mirada y el movimiento corporal) y el estilo atribucional (que es la forma como se explica la conducta de otras personas) (Pelphery, Adolphs \& Morris, 2004). Para otros autores es definida como la habilidad cognitiva que poseen los individuos para hacer una lectura de las emociones, creencias, deseos e intenciones de las demás personas y los propios, permitiendo realizar inferencias de posibles comportamientos a futuro (Flavell \& Miller, 1998; Wilson y Sperber, 2002).

La cognición social cuenta con una diversa cantidad de procesos que buscan comprender de manera específica el comportamiento de los seres humanos, cada uno de los cuales se centra en un área particular. Algunos de estos procesos son la identificación, expresión y manejo de emociones, la teoría de la mente (ToM), la empatía, percepción del otro y finalmente los procesos sobre el sí mismo (self). Por lo expuesto, es posible plantear que el campo de la cognición social busca comprender y explicar la influencia que tiene sobre un sujeto, ya sea de manera real o imaginada, las emociones, conductas y pensamientos de los individuos. Asimismo, el grado de intervención de la motivación y emoción, sobre la conducta y la cognición.

Los estudios emprendidos por algunos investigadores como Anderson, Bechara, Tranel y Damasio (1999) (Adolphs, Tranel y Damasio, 1998; citados por Grande-García, 2009) con pacientes que presentan daño cerebral, permitieron concluir que existen algunas áreas cerebrales que determinan la percepción social, cognición, toma de decisiones y juicios sociales. Algunas de estas áreas son: ventromedial, orbitofrontal y prefrontal, la amígdala y la corteza somatosensorial. Adicionalmente, Adolphs (1999) (citado por Grande-García, 2009) propone que el mundo social y las relaciones sociales establecidas por los individuos, se generan a partir del tipo de cognición que presenten. La cognición para este autor obedece de la ejecución de complejas redes neuronales, involucradas en la percepción, valoración, reconocimiento de los estímulos circundantes, las cuales constituyen cierto dispositivo para la formación de las representaciones del entorno social y por ende, de los congéneres. 
Los estudios realizados por Davidson, Putman \& Larson (2000), proponen que las personas con alteraciones en las estructuras cerebrales relacionados con el procesamiento de las emociones, tienen una mayor probabilidad de generar conductas violentas y agresivas. Estos autores también plantean que alteraciones en la zona prefrontal y corteza orbitofrontal, tienen como resultado una conducta por lo general de tipo impulsiva y agresiva.

Frente a lo expuesto, Damasio (2004) manifiesta que las emociones son básicamente el resultado de un proceso de evaluación mental que puede llegar a ser simple o complejo, y que traen consigo respuestas dirigidas hacia el cuerpo (estados corporales) y el cerebro (cambios mentales). A partir de lo expuesto, es posible inferir que una alteración en el proceso de evaluación mental del contexto puede tener como consecuencia esquemas cognitivos y respuestas motoras adversas al contexto social en el que se encuentra inmerso el individuo.

A partir de lo anterior, el objetivo de este artículo es ser un aporte a nivel empírico y conceptual desde la neurocriminología al sistema penal colombiano como medio probatorio frente a la toma de decisiones, políticas criminales, estrategias de prevención y promoción, así como la generación de programas de intervención centrados en las necesidades con victimarios desde el área penitenciaria. Se espera que el alcance de los resultados contribuya a seguir ampliando el marco de referencia conceptual y teórico desde la neurcriminología para el abordaje de la violencia de pareja.

\section{Método}

\section{Diseño}

Estudio ex post facto, transeccional de tipo correlacional.

\section{Participantes}

Para la selección de los participantes se utilizó un muestreo no probabilístico incidental, en el cual participaron 34 personas (17 hombres maltratadores y 17 hombres no maltratadores).

\section{Instrumentos y procedimiento}

\section{Pruebas visuales de procesamiento emocional}

Estas pruebas miden la habilidad para reconocer a través de la mirada o la expresión facial, el estado mental de una persona. Esta capacidad hace énfasis en el campo visuoperceptivo, lo que permite disminuir "la demanda de memoria, de funciones ejecutivas y contextual" (Ozonoff, 1991; BaronCohen, 1997; citados por De Achaval, 2010, p. 13). Derivada de este tipo de pruebas se describen a continuación los test elegidos.

\section{Test de reconocimiento emocional en caras (Baron-Cohen, 1997; citado por De Achaval, 2010)}

El test se estructura a partir de 20 fotografías o láminas, las cuales presentan expresiones faciales efectuadas por un individuo. Estas láminas se caracterizan por poseer unas condiciones de iluminosidad controladas y ajustadas, que siempre evidencian su rostro de frente. La persona manifiesta 10 emociones básicas y 10 emociones complejas, las cuales se encuentran plasmadas a través de fotografías impresas en color blanco y negro, con un diámetro de $10 "$ × 8 ".

Al aplicar la prueba, se le indica a la persona las siguientes instrucciones: "por favor elija la palabra que mejor describa lo que la persona está pensando o sintiendo. Debe responder lo más rápido posible. Si ninguno de los términos le parece del todo correcto, de todas maneras debe elegir uno de los dos términos".

\section{Test de reconocimiento emocional en ojos (Baron-Cohen, 2001; citado por De Achaval, 2010)}

La prueba se estructura en 36 fotografías o láminas que representan ojos de diferentes personas. Estas son exhibidas una por una a través de un orden fijo. Las láminas tienen plasmadas miradas que son 
estímulos que contienen a su alrededor cuatro palabras que representan emociones. La tarea del examinado consiste en elegir la palabra que mejor describe lo que la persona en la foto está pensando o sintiendo. Asimismo, se le entrega al examinado un glosario con todas las palabras y se le indica que puede revisar el listado en el momento que desconozca el significado de alguna de ellas, o el examinado le puede solicitar al evaluador que diga en voz alta el significado de la palabra. Se otorga un punto por cada acierto; el puntaje máximo de la prueba es de 36.

Test de faux pas (Gregory, et al., 2002; De Achaval, 2010)

Esta prueba mide la capacidad para identificar cuando un individuo dice algo inadecuado pero sin premeditarlo, toda vez que dicha acción era o podría haber tenido como consecuencia herir a otra persona.

El test se estructura a partir de 20 historias descritas por Gregory et al. (2002; citado por De Achaval, 2010) basadas en la metodología empleada por Stone et al. (1998; citado por De Achaval, 2010). La mitad de la prueba, 10 historias, se caracterizan por ser historias control, las cuales expresan un conflicto menor dentro de ellas, y que de acuerdo a los autores no constituye un faux pas, la otra mitad contiene un faux pas social, es decir, un paso en falso (metida de pata).

El examinador debe presentar y leer en voz alta y frente al sujeto, las historias solo una vez, en un orden determinado de manera fija y cada historia en una hoja independiente.

La prueba se encuentra estructurada con preguntas que permiten identificar la comprensión de detalles de la historia, es decir, preguntas con componente memorístico, al igual que preguntas que detectan faux pas. El sistema de calificación es el realizado por Stone et al. (1998; citado por De Achaval, 2010).
Test de historias ToM (Happé et al., 1999; citado por De Achaval, 2010)

La prueba se estructura a partir de 16 historias, las cuales fueron descritas por Happé et al. (1999, 2001; citado por De Achaval, 2010). La mitad de las historias evalúan la teoría de la mente (ToM), la otra mitad se caracteriza por ser historias físicas.

Al aplicarse la prueba, se le indica a la persona las siguientes instrucciones: "en cada página encontrará una historia para leer. Una vez que haya leído y entendido la historia, dé vuelta a la página. Hay una pregunta después de cada historia y quisiera que la responda. No vuelva a mirar la historia, así que antes de dar vuelta a la página asegúrese que haya entendido".

El sistema de puntuación para la calificación de la prueba se sustenta en los principios utilizados por Happé, es decir, al examinado se le otorgan 2 puntos por cada respuesta correcta, 1 punto por respuesta parcial o implícita, y ningún punto por respuesta incorrecta (de Achaval, 2010).

Variables: los dos test mencionados con anterioridad, se utilizaron dentro de la presente investigación para la evaluación de la Teoría de la Mente.

\section{EQ-Cambridge}

El EQ es un test que evalúa a través de 60 preguntas la respuesta empática del individuo frente a algunas situaciones planteadas. Las 60 preguntas se encuentran divididas en dos tipos: 40 preguntas que miden la respuesta empática y 20 preguntas de distracción.

La puntuación del test se realiza de la siguiente manera: 1 punto si el evaluado registra el comportamiento empático de manera cercana, o 2 puntos si el entrevistado registra el comportamiento fuertemente. Los reactivos se presentan de manera aleatoria. El EQ tiene un formato de elección auto o heteroaplicado.

El EQ no concibe como elementos separados las categorías afectivas y cognitivas, toda vez que estos 
son componentes que co-ocurren y no se pueden separar fácilmente. Por ello, abandona los postulados planteados en el que se separan dichas variables.

Variable: el test de EQ-Cambridge evalúa empatía, la cual permite comprender lo que otra persona está sintiendo o podría estar pensando, de tal manera que fortalece la capacidad de entender las intenciones de otros para poder predecir su comportamiento; lo que motiva la interacción efectiva de las personas con el mundo social que los rodea (Baron-Cohen \& Wheelwright, 2004). Pese a esta aproximación, es un concepto con diversas definiciones, entre ellas las que se centran en enfoques de tipo cognitivo y las de enfoques de tipo afectivo; sin embargo, los dos enfoques son esenciales para definir la empatía y por tanto no deben separarse. Por su parte el enfoque afectivo define la empatía como la respuesta emocional del observador en el estado afectivo de otro y el enfoque cognitivo considera que la empatía implica la comprensión de los sentimientos del otro (Kohler, 1929).

\section{Consideraciones éticas}

La participación e inclusión de las personas en el estudio: se realizó a través del contacto de posibles participantes; se les explicó el estudio y se invitó a que hicieran parte de él de manera voluntaria. La aceptación se plasmó mediante un consentimiento informado, el cual se caracteriza por poseer las condiciones de participación al igual que la forma de devolución de resultados. Asimismo, se tuvieron en cuenta para el desarrollo del estudio los lineamientos del informe Belmont, código deontológico y bioético de psicología (Ley 1090 de 2006) al igual que directrices para el desarrollo de estudios biomédicos en seres humanos (CIOMS, 1982).

\section{Análisis de datos y tratamiento estadístico}

El tratamiento de los datos se realizó mediante el paquete estadístico SPSS 22. Se llevó a cabo un análisis descriptivo de las variables involucradas (edad, procedencia y nivel educativo, así como las variables objeto de estudio), se realizaron análisis estadísticos de normalidad y anormalidad, comparación de medias entre grupos, correlaciones bivariadas entre grupos y análisis descriptivo. Para el análisis de resultados en cada uno de los instrumentos se tuvo en cuenta la puntuación total en la muestra normativa y la puntuación por grupos. Las pruebas estadísticas utilizadas para el análisis fueron: la prueba U Mann Whitney y Spearman.

\section{Resultados}

La muestra estuvo conformada por 34 hombres distribuidos en dos grupos, el primer grupo de 17 hombres maltratadores y el segundo grupo de 17 hombres no maltratadores. Dentro del análisis de las características sociodemográficas, se encontró que las edades de los participantes oscilaron entre 25 y 58 años, con una edad media de 40.21 años (9.905).

A nivel educativo, la muestra estuvo distribuida así: 7 de los participantes habían cursado bachillerato incompleto, y equivalen al 20,6\% de la muestra; 5 cursaron bachillerato completo, y equivalen al 14,7 \% de la muestra; 18 hombres tenían formación técnica, equivalente al $52,9 \%$ de la población; 4 personas tienen nivel de formación profesional, que equivalen al $11,8 \%$ de la población.

En la tabla 1 es posible evidenciar los estadísticos descriptivos con respecto a las puntuaciones obtenidas en cada una de las pruebas, las cuales evalúan cognición social en el grupo de hombres maltratadores y en el grupo de hombres no maltratadores.

\section{Faux pas}

Para el análisis de resultados de esta prueba los datos se dividieron en dos grupos, por un lado se establecieron puntuaciones directas para aquellas preguntas control donde no existía "metidas de pata", a esta parte de la prueba se le denomino faux pas control (en adelante fauxcon). Por otra parte, se establecieron las puntuaciones directas para aquellas historias que contenían "metidas de pata" y que para el presente estudio se denominaron como experimentales (en adelante fauxexp). 
Tabla 1.

Estadísticos descriptivos cognición social.

\begin{tabular}{|c|c|c|c|c|c|}
\hline & $\mathrm{N}$ & Media & Desviación estándar & Media & Desviación estándar \\
\hline FAUXCON & 17 & 15,53 & 7,072 & 25,47 & 2,348 \\
\hline FAUXEXP & 17 & 30,24 & 21,661 & 72,18 & 4,876 \\
\hline HISTORIAS & 17 & 16,47 & 2,125 & 17,06 & 659 \\
\hline OJOS & 17 & 19,82 & 4,172 & 24,53 & 2,183 \\
\hline CARAS & 17 & 16,88 & 1,536 & 18,18 & 1,131 \\
\hline EQ & 17 & 65 & ,493 & ,41 &, 507 \\
\hline
\end{tabular}

Fuente: elaboración propia.

\section{Faux pas control}

En las preguntas del faux pas control, para la muestra los puntajes oscilaron entre 3 y 30 , con una media de 20,50 y una SD de 7,237.

La hipótesis nula para esta prueba fue la siguiente:

H0: la distribución de fauxcon es la misma entre las categorías de grupos.

Los puntajes obtenidos por el grupo de maltratadores osciló entre 3 y 26, con media 15,53 y una SD de 7,072 (tabla 1) y en el grupo de no maltratadores entre 22 y 30 , con una media de 25,47 y una SD de 2,348 (tabla 1). Para la validación de hipótesis se utilizó el estadístico U Mann-Whitney y Kolmogorov-Smirnov con los siguientes resultados: $U=0.000$ y $p=0.000$, razón por la cual se rechaza la hipótesis nula.

\section{Faux pas experimental}

En el mismo instrumento para las preguntas con faux pas o "metida de pata", para la muestra normativa los puntajes oscilaron entre 0 y 79 , con una media (con las derivaciones estándar entre paréntesis) de 51,21 (26,308).

La hipótesis nula para esta prueba fue la siguiente:

H0: la distribución de fauxexp es la misma entre las categorías de grupos.

Los puntajes obtenidos por el grupo de maltratadores osciló entre 0 y 79, con una media de 30,24 y una SD de 21,661 (tabla 1) y en el grupo de no maltratadores entre 64 y 79 , con una media de 72,18 y una SD de 4,876 (tabla 1). En la validación de hipótesis se obtuvieron los siguientes resultados $U=0.000$ y $p=0.000$, rechazando la hipótesis nula.

\section{Test de historias}

Los puntajes obtenidos en el test de historias para la muestra normativa, oscilaron entre 12 y 19 , con una media de 16,76 y una SD de 1,577 , respectivamente.

La hipótesis nula para esta prueba fue la siguiente:

HO: la distribución de historias es la misma entre las categorías de grupos.

Los puntajes obtenidos por el grupo de maltratadores osciló entre 12 y 19, con una media de 16,47 y una SD de 2,125 (tabla 1) y en el grupo de no maltratadores entre 16 y 18, con una media de 17.06 y una SD de 0.659 (tabla 1). En la validación de hipótesis se obtuvieron los siguientes resultados $U=$ 3.339 y $p=0.240$, razón por la cual se conserva la hipótesis nula.

\section{Test de la mirada (ojos)}

En el test de la mirada (ojos) los puntajes para la muestra normativa oscilaron entre 11 y 27 , con una media de 22,18 y una SD de 4,056.

La hipótesis nula para esta prueba fue la siguiente: 
H0: la distribución de ojos es la misma entre las categorías de grupos.

Los puntajes obtenidos por el grupo de maltratadores osciló entre 11 y 26 , con una media de 19,82 y una SD de 4,172 (tabla 1) y en el grupo de no maltratadores entre 18 y 27 , con una media de 24,53 y una SD de 2,183 (tabla 1). En la validación de hipótesis se obtuvieron los siguientes resultados $U=0.001$ y $p=0.002$, razón por la cual se rechaza la hipótesis nula.

\section{Test de caras}

Los resultados obtenidos en el test de caras para la muestra normativa oscilaron entre 13 y 19, con una media de 17,53 y una SD de 1,482.

La hipótesis nula para esta prueba fue la siguiente:

HO: la distribución de caras es la misma entre las categorías de grupos.

Los puntajes obtenidos por el grupo de maltratadores osciló entre 13 y 18, con una media de 16,88 y una SD de 1,536 (tabla 1) y en el grupo de no maltratadores entre 15 y 19, con una media de 18,18 y una SD de 1,131 (tabla 1). En la validación de hipótesis se obtuvieron los siguientes resultados $U=0.004$ y $p=0.017$, razón por la cual se rechaza la hipótesis nula.

\section{EQ de Cambridge}

En el EQ de Cambridge para la muestra, las puntuaciones oscilaron entre 0 y 1 , con una media de 0,53 y una SD de 0,507 .

La hipótesis nula para esta prueba fue la siguiente:

HO: la distribución de EQ es la misma entre las categorías de grupos.

Los puntajes obtenidos por el grupo de maltratadores osciló entre 0 y 1 , con una media de 0,65 y una SD de 0,493 (tabla 1) y en el grupo de no maltratadores entre 0 y 1 , con una media de 0,41 y una SD de 0,507 (tabla 1). En la validación de hipótesis se obtuvieron los siguientes resultados $U=0.245$ y $p=$ 0.734 , razón por la cual se acepta la hipótesis nula.

\section{Discusión y conclusiones}

En los resultados obtenidos en las tareas que evalúan cognición social en la comparación de medias, se encontró que instrumentos como el faux pas, el test de la mirada, test de caras, refieren que la distribución de los puntajes no es la misma para los dos grupos, y por tanto, existe la probabilidad que los hombres maltratadores evaluados identifiquen situaciones como altamente conflictivas aunque no lo sean, caso contrario ocurre en los hombres no maltratadores, donde las puntuaciones superiores indican una diferenciación más clara entre aquellos hechos que no generan conflicto.

Asimismo, los resultados permiten mencionar que el grupo de hombres maltratadores evaluados presenta ciertas dificultades para la identificación de estados mentales complejos; es decir, identificar, reconocer y atribuir intenciones o estados emocionales en otras personas, situación que interfiere en la interacción positiva con las personas de su entorno cercano, específicamente su pareja, para el caso de las personas evaluadas.

Estas características, de acuerdo a Vitaro, Brendgen y Tremblay (2002), se deben a que la agresión de tipo impulsiva se caracteriza por ser una respuesta inmediata ante situaciones reales o imaginadas, que se perciben como amenazantes o de provocación, las cuales son generadas por un alto componente emocional. Postulado que también ha sido investigado por Raine (2002), quien concluyó que los sujetos con una alteración en el lóbulo frontal responden de manera agresiva ante estímulos de tipo trivial; esta última afirmación debe ser validada para el caso de las personas evaluadas mediante estudios especializados que permitan aportar evidencia al respecto.

La distribución de los puntajes en el test de historias y el EQ de Cambridge, fue similar en los dos grupos, y por tanto se considera que los participantes de la presente investigación poseen la capacidad para inferir estados mentales a partir 
de las intenciones de los personajes, así como la capacidad para interpretar y reconocer los sentimientos y emociones en el otro. Sin embargo, estos resultados podrían relacionarse con un factor de deseabilidad social que puede encontrarse presente en los dos grupos, principalmente en el de hombres maltratadores, quienes pueden identificar las emociones del otro como un mecanismo de acercamiento y desarrollo de la aceptación social (Lawrence et al., 2004).

Estos resultados podrían explicarse en el caso de los maltratadores acorde a lo expuesto por Andreu (2009), quien ha desarrollado investigaciones relacionadas con la agresión premeditada, que se caracteriza porque su objetivo no solo es causar daño, sino también alcanzar un propósito; debido a ello, no se produce en respuesta a una acción percibida como amenazante o de provocación, y tampoco requiere de una activación emocional alta. En este sentido, la explicación de la violencia de pareja se podría postular desde la cognición social en dos modalidades, mediante la agresión impulsiva y premeditada, es decir, que no hay solo un tipo de agresión presente en el grupo de hombres maltratadores evaluados, siendo este un aporte importante para la comprensión de esta problemática desde el modelo de clasificación bimodal en agresores propuesto por Chase, O'Leary \& Heyman (2001), toda vez que permitirá realizar intervenciones de acuerdo a la etiología de la conducta agresora, la incidencia en el campo legal y clínico, así como la comprensión de aquellas circunstancias que motivan la reincidencia de este tipo de comportamientos.

Si bien es cierto, cada uno de los hallazgos generan alternativas para la compresión de la violencia de pareja, es evidente que esta no puede ni debe ser explicada únicamente por un modelo unicausal, y por tanto, para abordarla de manera directa y generar un efecto en las estrategias de mitigación y prevención, es necesario crear herramientas efectivas, pero a la vez reconocer las personas a quien vaya dirigido, puesto que el desconocimiento de las características de la población podrían estar generando el bajo impacto en los programas de intervención y planeación que han sido practicados en la actualidad.
En general, fue posible evidenciar que existe un mejor desempeño en las tareas que evalúan cognición social en el grupo de hombres no maltratadores respecto al grupo de hombres maltratadores; aspectos que facilitan la interacción de estas personas con el medio social en que se desenvuelve, y favorecen una interpretación adecuada a la realidad, permitiendo que generen respuestas adaptativas ante las situaciones que les generen malestar.

Si bien es cierto, los resultados de la presente investigación, así como otras investigaciones realizadas, podrían generar una interpretación de la violencia de pareja desde la agresión impulsiva y premeditada, es necesario reiterar que esta problemática no puede ser explicada por un modelo unicausal, sino que para su comprensión se deben tener en cuenta las relaciones que existen a nivel genético, neurobiológico, psicofisiológico y social con este tipo de comportamientos.

Asimismo, este tipo de investigación resulta ser un aporte, tal vez modesto pero sin duda promisorio, para contribuir no solo a la construcción de marcos de referencia que posibiliten y orienten la elaboración de políticas criminales y públicas, donde no solo se tengan en cuenta las características de la agresión premeditada e impulsiva, sino que también posibilita abrir el camino para el cambio de los paradigmas en el tratamiento y la toma de decisiones por parte de los jueces, al reconocer la influencia del factor neuropsicológico en la etiología de la conducta agresora desde la perspectiva del procesamiento emocional.

Los hallazgos del presente estudio conllevan a plantear la necesidad de incluir en las valoraciones de los agresores por el delito de violencia de pareja, la variable de procesamiento emocional como medio probatorio, con el fin de determinar no solo la influencia que pueda llegar a tener el lóbulo frontal en el procesamiento de la información, y por ende, el desarrollo de las conductas punibles, sino también poder realizar la planeación del posible tratamiento, estimación del riesgo de reincidencia del individuo y nivel de peligrosidad, siendo esta última premisa un aporte importante para la victimología. 
En lo relacionado al campo forense se realiza un aporte significativo desde la neurocriminología, que permite seguir consolidando un marco empírico para la comprensión de las conductas violentas por parte de hombres agresores hacia sus parejas y por ende, un marco conceptual de orientación para los operadores judiciales al igual que la generación de políticas criminales.

Finalmente, los resultados del presente estudio evidencian la pertinencia de realizar evaluaciones no solo psicológicas sino también neuropsicológicas en el campo judicial, las cuales permitan analizar las dimensiones cognitivas y conductuales de los agresores, facilitando de esta forma la comprensión de los factores etiológicos, y con ello la realización de intervenciones de la conducta agresora ajustadas a la necesidad del sujeto.

\section{Referencias}

Adolphs, R. (2001). The neurobiology of social cognition. Current Opinion in Neurobiology, 11, 231-239.

Andreu, J.M. (2009). Propuesta de un modelo integrador de la agresividad impulsiva y premeditada en función de sus bases motivacionales y socio-cognitivas. Psicopatología Clínica Legal y Forense, 9, 85-98.

Amor, P.J., Echeburúa, E. y Loinaz, I. (2009). ¿Se puede establecer una clasificación tipológica de los hombres violentos contra su pareja? Journal of Clinical and Health Psychology, 9(3), 519-539.

Amor, P.J., Bohórquez, I.A. y Echeburúa, E. (2006). ¿Por qué y a qué coste físico y psicológico permanece la mujer junto a su pareja maltratadora? Acción Psicológica, 2, 129-154.

Barón, J. (2010). La violencia de pareja en Colombia y sus regiones. Banco de la República, (128).

Baron-Cohen, S. \& Wheelwright, S. (2004). The empathy quotient: An investigation of adults with asperger syndrome or high functioning autism, and normal sex differences. Journal of Autism and Developmental Disorders, 34(2), 163-175.

Bartholomew, K., Regan, K.V., Oram, D.O. \& White, M.A. (2008). Correlates of partner abuse in male same-sex relationships. Violence and Victims, 23, 344-360.

Butman, J. (2001). La cognición social y la corteza cerebral. Revista Neurológica Argentina, 26, 117-122.

Burke, T.W., Jordan, M.L. \& Owen, S.S. (2002). A cross-national comparison of gay and lesbian domestic violence. Journal of Contemporary Criminal Justice, 18, 231-257.

Burke, L. K. \& Follingstad, D.R. (1999). Violence in lesbian and gay relationships: Theory, prevalence, and correlational factors. Clinical Psychology Review, 19, 487-512.

Carreño, P.A. (2011). Comportamiento de la violencia intrafamiliar, Colombia. Bogotá: Instituto Nacional de Medicina Legal y Ciencias Forenses.

Congreso de la República de Colombia. (2006). Ley 1090. Por la cual se reglamenta el ejercicio de la profesión de Psicología, se dicta el Código Deontológico y Bioético y otras disposiciones. Diario Oficial 46.383 del 6 de septiembre de 2006.

Congreso de la República de Colombia. (2000). Ley 599 de 2000. Por la cual se expide el código penal.

Chase, K.A., O’Leary, K.D. \& Heyman, R. (2001). Categorizing partner-violent men within the reactive-proactive typology model. Journal of Consulting and Clinical Psychology, 69, 567-572.

Damasio, A. (2004). El error de Descartes. La emoción, la razón y el cerebro humano. Barcelona: Crítica.

Davidson, R., Putman, K. \& Larson, C. (2000). Dysfunction in the neural circuitry of emotion regulation. A possible prelude to violence. Science, (289), 591-594. 
Decety, J. (2010). The neurodevelopment of empathy in humans. Developmental Neuroscience, 32(4), 257-267. doi.org/10.1159/000317771.

Decety, J. (2008). Who caused the pain? A functional MRI investigation of empathy and intentionality in children. Neuropsychologia, 46, 2607-2614.

Decety, J. \& Meyer, M. (2008). From emotion resonance to empathic understanding: a social developmental neuroscience account. Dev Psychopathol, 20(4), 1053-80. doi: 10.1017/ S0954579408000503.

De Achaval, D. (2010). Evaluación de la cognición social en pacientes con esquizofrenia y sus familiares de primer grado no afectados. Buenos Aires: Universidad de Belgrano.

Flavell, J. H. \& Miller, P. (1998). Social cognition. In W. Damon, D. Kuhn \& R.S. Siegler (eds.), Handbook of Child Psychology. Cognition, perception and language 3 (pp. 851-898). New York: Wiley \& Sons.

Grande-García, I. (2009). Neurociencia social: el maridaje entre la psicología social y las neurociencias cognitivas. Revisión e introducción a una nueva disciplina. Revista anales de psicología, 25(1), 1-20.

Greely, T. (2008). Neuroscience and criminal justice: Not responsibility but treatment. Kansas Law Review, 56, 11-38. Retrieved from https:// law.ku.edu/sites/law.drupal.ku.edu/files/docs/ law_review/v56/04-Greely_Final.pdf.

Gregory, C., Lough, S., Stone, V., Erzinclioglu, S., Martin, L., Baron-Cohen, S. \& Hodges, J.R. (2002). Theory of mind in patients with frontal variant frontotemporal dementia and Alzheimer's disease: Theoretical and practical implications. Brain, 125(4), 752-64.

Henry, S. \& Plemmons, D. (2012). Neuroscience, neuropolitics and neuroethics: The complex case of crime, deception and FMRI. Sci Eng Ethics, 18(3), 573-91. doi: 10.1007/s11948-012-9393-4.
Instituto Colombiano de Medicina Legal y Ciencias Forenses. (2015). Forensis: Datos para la vida, 16(1).

Jouriles, E.N., McDonald, R., Norwood, W. D. \& Ezell, E. (2001). Domestic violence in the lives of children. Washington, D.C.: Graham y Edleson S.A.

Kohler, W. (1929). Gestalt psychology. New York: Liveright.

Krug, E.G., Dahlberg, L.L., Mercy, J.A., Zwi, A.B. \& Lozano, R., (2002). World report on violence and health. Ginebra: Organización Mundial de la Salud. Retrieved from http://www.unwomen. org/es/what-we-do/ending-violence-againstwomen/facts-and-figures\#sthash.LUoOnxMC. dpuf.

Lawrence, E.J., Shaw, P., Baker, D., Baron-Cohen, S. \& David, A.S. (2004). Measuring empathy: Reliability and validity of the empathy quotient. Psychological Medicine, 34, 911-924. doi: 10.1017/S0033291703001624.

Morse, B. (1995). Beyond the conflict tactics scale: Assessing gender differences in partner violence. Violence and Victims, 10, 251-272.

Olaya, B., Tarragona, M.J., de la Osa, N. y Ezpeleta, L. (2008). Protocolo de evaluación de niños y adolescentes víctimas de la violencia doméstica. Papeles del psicólogo, 29(1), 123-135.

Organización Mundial de la Salud. (2002). Informe mundial sobre la violencia y la salud. Francia.

Consejo de Organizaciones Internacionales de las Ciencias Médicas (CIOMS), Organización Mundial de la Salud. (2002). Pautas éticas internacionales para la investigación biomédica en seres humanos. Recuperado de: http:// www.ub.edu/rceue/archivos/ Pautas_Eticas_ Internac.pdf.

Pelphery, K., Adolphs, R. \& Morris, J.P. (2004). Neuroanatomical substrates of social cognition dysfunction in autism. Mental retardations and developmental disabilities, 10, 259-271. 
Procuraduría General de la República, República Dominicana. (2005). Los lineamientos de la política criminal dominicana, dentro de los parámetros del Plan Nacional de Seguridad Democrática para la República Dominicana. Ciudad:

Raine, A. (2002). Psicopatía, violencia y neuroimagen. En A. Raine, J. Sanmartín (eds.)., Violencia y psicopatía (pp. 59-88). Barcelona: Ariel.

Ribero, R. y Sánchez, F. (2004). Determinantes, efectos y costos de la violencia intrafamiliar en Colombia. Documento CEDE 2004-44, Bogotá: Universidad de los Andes.

Straus, M.A. (1993). Physical assaults by wives: A major social problem. En R.J. Gelles \& D. Loseke (eds.). Current controversies on family violence, 67-87. Newbury Park, C.A.: Sage.

Straus, M.A. \& Gelles, R. J. (1988). How violent are American families? Estimates from the National Family Violence Resurvey and other studies.
Family abuse and its consequences: New directions in research, 14-36.

Turell, S. C. (2000). A descriptive analysis of samesex relationship violence for a diverse sample. Journal of Family Violence, 15, 281-293.

Vitaro, F., Brendgen, M. \& Tremblay, R.E. (2002). Reactively and proactively aggressive children: antecedents and subsequent characteristics. Journal of Child Psychology and Psychiatry, 43, 495-505.

Uribe, V. C. (2010). Una breve introducción a la cognición social: procesos y estructuras relacionados. Revista Con-texto, 4, 1-10.

Urrego, Y. (2011) Cognición social y trauma temprano. Boletín Electrónico de Salud Escolar, TIPIC Universidad Piloto de Colombia, 7(2).

Wilson, D. \& Sperber, D. (2002). Relevance theory. En L. Horn \& G. Ward (eds.), Handbook of Pragmatics Psycholinguistics (pp. 34-78). Oxford: Blackwell. 